\title{
Atomic scale environmental transmission electron microscopy study of the surface mobility of ceria nanocubes
}

\author{
M. Bugnet ${ }^{1}$, S. H. Overbury ${ }^{2}$, Z. Wu ${ }^{2}$, F. J. C. S. Aires ${ }^{3}$ and T. Epicier ${ }^{1}$ \\ 1. University of Lyon, INSA-Lyon, UCBL Lyon 1, MATEIS, UMR 5510 CNRS, 69621 Villeurbanne \\ Cedex, France. \\ 2. Chemical Science Division, Center for Nanophase Materials Science, Oak Ridge National Laboratory, \\ Oak Ridge, Tennessee 37831, USA. \\ 3. University of Lyon, UCBL Lyon 1, IRCELYON, UMR 5256 CNRS, 69100 Villeurbanne, France.
}

Transmission electron microscopy (TEM) is a well-established characterization technique to combine bulk as well as surface analysis in a wide range of materials at the nanoscale. Since the large majority of materials are not utilized in moderate or high vacuum (HV), investigating them in situ in the TEM in more realistic environmental conditions appears necessary to understand fundamental structural and chemical aspects at surfaces in several fields of research such as catalysis, corrosion, or crystal growth. In this respect, control of the nature and the pressure of the atmosphere surrounding the specimen while allowing nanoscale down to atomic resolution analysis in a TEM is of great importance.

$\mathrm{CeO}_{2}$ is a fundamentally interesting and technologically important catalyst and catalyst support [1]. With a strong tendency to be reduced under the electron beam, $\mathrm{CeO}_{2}$ is also a challenging material in a regular HV TEM column, and offers a perfect field of play for redox state control in the ETEM. The redox properties of $\mathrm{CeO}_{2}$ have been widely studied in the ETEM using electron energy-loss spectroscopy (EELS), shedding light on the crystal structure and chemistry [2 - 4]. Beyond tuning the redox state, indepth understanding of the surface atomic structure and atomic mobility of catalytically active surfaces is of primary importance in terms of surface reactivity. Nevertheless, in depth atomic scale surface analysis in environmental conditions is lacking. In the present work, the atomic mobility at surfaces induced by the partial pressure and temperature is studied on $\mathrm{CeO}_{2}$ nanocubes [5] within a dedicated FEI Titan 80-300 Cs-image corrected environmental TEM (ETEM), equipped with a high-speed CCD Gatan OneView $^{\text {TM }}$.

The mobility of $\mathrm{Ce}$ and $\mathrm{O}$ atoms is monitored using HRTEM under various atmospheres, at shown in Figure 1. In thin areas, assuming kinematic conditions, the intensity of "black atoms" atomic columns in HRTEM images can be taken as proportional to the inverse of the column occupancy. Using fast recording of atomic resolution images ( $25 \mathrm{fps}$ ), the mobility of Ce atoms is directly observed. A scheme was subsequently developed to track and quantify the intensity of Ce columns, and thus the mobility of $\mathrm{Ce}$ atoms, as highlighted in figure 2. These results demonstrate that the mobility of $\mathrm{Ce}$ atoms on (001) surfaces under HV conditions is relatively high, with notable variations from the centre of the edge to the corner of the nanocube (see figure 1, top). Furthermore, the mobility decreases when the atmosphere is changed from $\mathrm{HV}$ to $\mathrm{O}_{2}$ partial pressure, then decreases even further when $\mathrm{CO}_{2}$ is adsorbed on the surface, as confirmed by EELS. This work highlights that the (100) surface mobility of $\mathrm{CeO}_{2}$ can be tuned under different atmospheres, and that fine effects can be monitored through the analysis of atomic column intensities. The effect of temperature on the surface mobility under $\mathrm{HV}, \mathrm{O}_{2}$ and $\mathrm{CO}_{2}$ atmosphere will be presented. This work opens a field of study for direct visualization and control of atomic scale phenomena at surfaces such as carbonate adsorption and desorption. The current capabilities and limits of this approach in the ETEM will be discussed [6]. 
References:

[1] A. Trovarelli, "Catalysis by Ceria and Related Materials", Imperial College Press, London (2002).

[2] R. Wang et al, Nano Lett. 8 (2008) 962.

[3] F. J. C. S. Aires et al, EMC 2016: Proceedings, Wiley-VCH Verlag GmbH \& Co.

[4] T. Epicier et al, to appear in J. of Microscopy, special issue (2017).

[5] Z. Wu et al, Langmuir 26 (2010), p. 16595.

[6] M. B. acknowledges funding from INSA Lyon through a BQR project THERMOS. S. H. O. and Z. W. are supported by the U.S. Department of Energy, Office of Science, Office of Basic Energy

Sciences, Chemical Sciences, Geosciences, and Biosciences Division. The authors acknowledge M. Aouine for assistance in TEM preparation. The electron microscopy work presented here has been performed on a FEI Titan ETEM at the CLYM: Lyon-St-Etienne centre for electron microscopy.

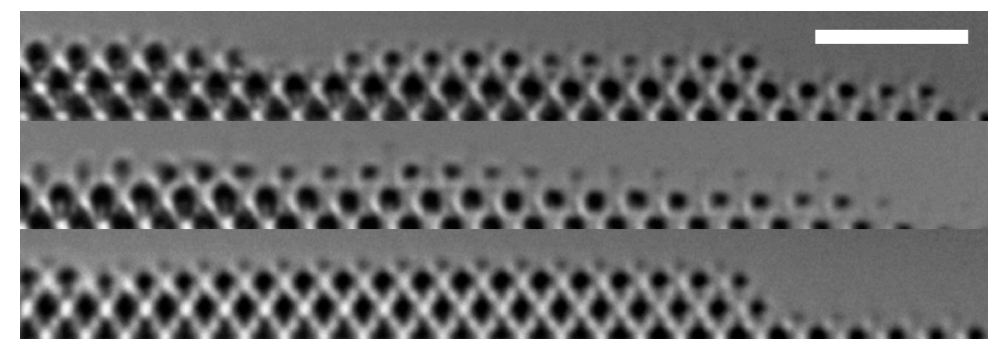

Figure 1. (001) surface of a ceria nanocube observed along [110] in (from top to bottom): $\mathrm{O}_{2}\left(510^{-2}\right.$ mbar), high vacuum (5 $\left.10^{-6} \mathrm{mbar}\right)$ and $\mathrm{CO}_{2}\left(2.510^{-2} \mathrm{mbar}\right)$. Each 'dark atoms' image is averaged over 2 seconds according to original micrographs recorded at $25 \mathrm{fps}$. This procedure shows that the terminating layer is mainly constituted by $\mathrm{O}$ atoms with some mobility under $\mathrm{O}_{2}, \mathrm{Ce}$ atoms with a great mobility under high vacuum and $\mathrm{O}_{2}$, and without any mobility under $\mathrm{CO}_{2}\left(300 \mathrm{kV}, 20^{\circ} \mathrm{C}\right)$. Scale bar: $1 \mathrm{~nm}$.

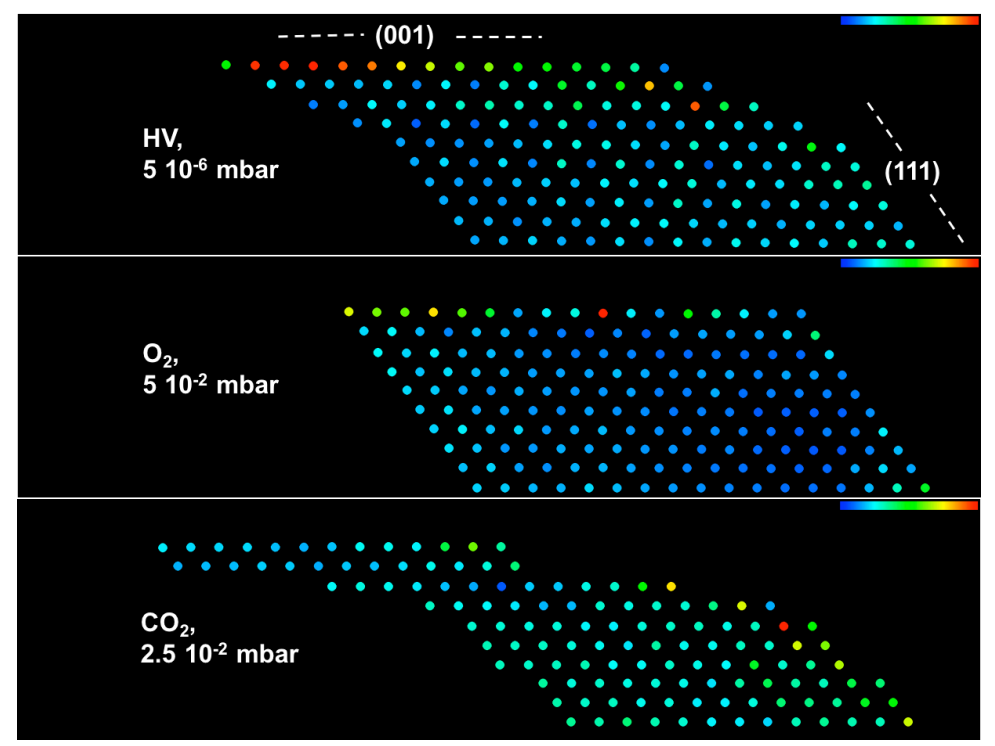

Figure 2. Variation of Ce surface mobility at (001) and (111) surfaces of a ceria nanocube $(300 \mathrm{kV}$, $20^{\circ} \mathrm{C}$ ). Each dot is placed at a single atomic column position, and its color indicates the variation of intensity over 150 images recorded at $25 \mathrm{fps}$ (Red: high variation; Blue: low variation). For the (001) surface, the column intensities vary strongly under high vacuum, indicating a high mobility (red). The mobility is lower under $\mathrm{O}_{2}$ atmosphere, and nearly stops when $\mathrm{CO}_{2}$ is adsorbed (blue). The mobility of Ce atoms on (111) surfaces is relatively low independently of the conditions. 